\title{
Divergência genética entre genótipos de pimenta com base em caracteres morfo-agrônomicos
}

\section{Luis Felipe V Ferrão ${ }^{1}$; Paulo Roberto Cecon ${ }^{1}$; Fernando Luis Finger²; Fabyano F e Silva ${ }^{1}$; Mario Puiatti ${ }^{2}$}

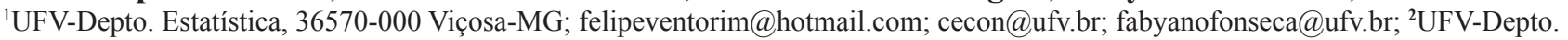
Fitotecnia; ffinger@ufv.br; mpuiatti@ufv.br

\section{RESUMO}

O gênero Capsicum compreende um grupo altamente diversificado de pimentas e pimentões constituído por grande número de espécies. A caracterização dos materiais existentes quanto à divergência genética torna-se de importância fundamental visando trabalhos de melhoramento. Técnicas multivariadas foram utilizadas para avaliar a divergência genética entre 34 subamostras da coleção de germoplasma de Capsicum baccatum da Universidade Federal de Viçosa. Foram utilizados cinco descritores quantitativos propostos pelo International Plant Genetic Resources Institute, em um experimento conduzido em condições de campo, em Viçosa-MG, no delineamento de blocos ao acaso. A divergência genética entre os tratamentos foi determinada pelas técnicas multivariadas, baseadas na análise de agrupamento e de variáveis canônicas. As variáveis analisadas foram matéria fresca do fruto, comprimento do fruto, espessura do pericarpo, número de sementes por frutos e teor de sólidos solúveis. Houve diferença significativa entre as subamostras para todos os descritores avaliados. Observou-se concordância entre as técnicas multivariadas utilizadas e foi possível separar as subamostras em cinco grupos distintos. As subamostras BGH 1739 e BGH 1646 se destacaram apresentando bom potencial para uso em programas de melhoramento, visando à obtenção de bons materiais para consumo in natura ou para industrialização.

Palavras chave: Capsicum baccatum, germoplasma, análises multivariadas, melhoramento genético.

\section{ABSTRACT}

Genetic divergence among sub-samples of pepper based on morpho-agronomic characters

The genus Capsicum comprises a varied group of hot and sweet peppers with a large number of species. The characterization of materials for genetic divergence becomes of paramount importance for breeding programs. Multivariate techniques were used to evaluated the genetic divergence among 34 sub-samples of Capsicum baccatum peppers from the Horticultural Germplasm Bank from the Federal University of Viçosa. Five quantitative descriptors proposed by International Plant Genetic Resources Institute were utilized in a field experiment carried out in Viçosa, Minas Gerais State, Brazil, in a randomized block design. The genetic divergence among the subsamples was determined by cluster analysis and canonical variables. The variables fruit average weight, fruit length, fruit diameter, number of seeds per fruit and content of soluble solids were evaluated. There was significant difference among sub-samples for all descriptors evaluated. General agreement among all multivariate techniques used in the work was observed and it was possible to separate the sub-samples in five distinct groups. The sub-samples BGH 1739 and BGH 1646 stood out showing good potential for use in breeding programs aiming to produce good materials for fresh consumption or processing purposes.

Keywords: Capsicum baccatum, germplasm, multivariate techniques, breeding.

\section{(Recebido para publicação em 2 de junho de 2010; aceito em 29 de agosto de 2011)}

(Received on June 2, 2010; accepted on August 29, 2011)

$\mathrm{O}$ gênero Capsicum compreende um grupo altamente diversificado de pimentas e pimentões originado do continente Americano (Lannes et al., 2007), constituído por um grande número de espécies. Destas, apenas cinco são comercialmente cultivadas: Capsicum annuum, C. baccatum, $C$. chinense, C. frutescens e C. pubescens (Pickersgill, 1997).

As pimentas são uma cultura de grande importância sócio-econômica. Contribuem como fonte geradora de renda na pequena propriedade e na fixação de pessoas na área rural. Aliado a isso é uma cultura geradora de empregos diretos e indiretos. As grande agroindús- trias do ramo das pimentas, possuem extensas áreas de cultivo (próprias ou em parceria) empregando significativo número de pessoas, principalmente na época de plantio (Wagner, 2003). Tal fato faz com que pimentas e pimentões estejam entre as 10 hortaliças de maior importância econômica no mercado hortifrutigranjeiro nacional (Echer et al., 2002), tornando a cultura um dos bons exemplos de agricultura familiar e de interação pequeno agricultor-agroindústria (Embrapa Hortaliças, 2007). Com grande diversidade de cores, formatos e sabores; o mercado é bastante diversificado, indo desde a comercialização para consumo in natura e conservas caseiras, até a exportação de produtos industrializados.

Dentre as espécies cultivadas, a Capsicum baccatum é a mais consumida nas regiões Sul e Sudeste do país, sendo popularmente conhecida como dedo-de-moça ou cambuci (Embrapa Hortaliças, 2007). Representada por diferentes variedades botânicas, a $C$. baccatum var. baccatum e C. bacatum var. pendulum são empregadas na culinária de todo o mundo, por reunirem atributos importantes como aparência, textura, cor, aroma, teor de vitaminas e pungência.

A existência de variabilidade genética na população é condição básica para o sucesso em programa de melhoramento 
genético. Pickersgill (1997), estudando a importância dos recursos genéticos no melhoramento de Capsicum spp., concluiu que a diversidade disponível dentro das espécies domesticadas tem sido pouco explorada e ainda não foi esgotada. Dessa forma, os bancos de germoplasma são de suma importância, uma vez que colocam à disposição dos pesquisadores essa variabilidade (Gonçalves et al., 2008) que permite a seleção de genótipos superiores e possibilita o aumento da freqüência de alelos favoráveis. Entretanto, as subamostras conservadas nesses bancos são pouco utilizadas devido a uma série de dificuldades e deficiências, tais como a falta de documentação e de descrição adequada, e ainda, a insuficiente avaliação dos materiais genéticos dessas coleções, o que limita a ação de melhoristas (Gepts, 2006).

Uma das maneiras de estimar a diversidade genética é por meio de técnicas multivariadas, uma ferramenta que permite combinar as múltiplas informações contidas na unidade experimental, possibilitando a caracterização dos genótipos com base em um conjunto de variáveis. Diferentes procedimentos estatísticos podem ser utilizados, dentre eles, estão a distância generalizada de Mahalanobis, as variáveis canônicas e os componentes principais. A escolha do método é função da precisão desejada, da facilidade de análise e interpretação dos resultados e da forma de obtenção dos dados (Cruz \& Carneiro, 2006). Esses estudos normalmente são complementados pelos métodos aglomerativos e hierárquicos de agrupamento, como o método de Tocher (Rao, 1952) e média das distâncias (UPGMA) respectivamente.

O presente trabalho teve como objetivo avaliar a divergência genética entre subamostras de pimenta (Capsicum baccatum) do Banco de Germoplasma de Hortaliças (BGH) da Universidade Federal de Viçosa, por meio de técnicas multivariadas.

\section{MATERIAL E MÉTODOS}

Trinta e quatro subamostras de $C$. baccatum do Banco de Germoplasma (BGH) da Universidade Federal de Vi- çosa (UFV), proveniente de diferentes regiões (Tabela 1 ), foram cultivados a campo, durante o ano de 2008/09, na horta experimental do departamento de Fitotecnia da UFV (642 m altitude, $20^{\circ} 45^{\prime} \mathrm{S}$ e $\left.42^{\circ} 51^{\prime} \mathrm{W}\right)$. O experimento foi conduzido sob o delineamento experimental de blocos ao acaso com cinco repetições.

Cinco frutos maduros de cada unidade experimental foram coletados e segundo descritores do gênero Capsicum (IPGRI, 1995), foram analisados os caracteres morfo-agronômicos: matéria fresca do fruto (MF), comprimento do fruto $(\mathrm{CF})$, espessura do pericarpo (EP), número de sementes por frutos (SEM) e teor de sólidos solúveis (TSS). A medida da espessura do pericarpo (EP) foi realizada por meio de paquímetro utilizando apenas a porção mediana do fruto. Para medição do teor de sólidos solúveis dos frutos (TSS) utilizou-se um refratômetro do tipo Abbé, expresso em grau Brix. O pericarpo de cada fruto fresco foi macerado, sendo recolhida uma amostra do suco de cada um; esta foi aplicada sobre a superfície luminosa do refratômetro para realização da leitura.

A existência de variabilidade genética entre as subamostras foi testada por meio da análise de variância utilizando o teste $\mathrm{F}$, a $5 \%$ de probabilidade. As médias foram posteriormente agrupadas pelo critério de Scott-Knott a 5\% de probabilidade.

A divergência genética entre os tratamentos foi determinada pelas técnicas multivariadas, baseadas na análise de agrupamento e de variáveis canônicas. $\mathrm{Na}$ análise de agrupamento, utilizou-se a distância generalizada de Mahalanobis $\left(\mathrm{D}^{2}\right)$ como medida de dissimilaridade e na formação dos grupos, sendo o agrupamento das subamostras realizado pelo método hierárquico UPGMA, citado por Rao (1952). A contribuição relativa das variáveis para a divergência genética foi determinada utilizando o critério proposto por Singh (1981).

Segundo Cruz \& Carneiro (2006), esse método de agrupamento pode envolver matrizes de dissimilaridade de ordem elevada e o agrupamento realizado pode provocar perda de informações do grau de dissimilaridade. Dessa forma, a dissimilaridade genética também foi representada por dispersão dos escores em gráficos empregando a técnica de variáveis canônicas, em que os eixos foram representados pelas primeiras variáveis canônicas, que possuíam a maior porcentagem da variância total envolvendo os caracteres estudados.

Com base nas informações apresentadas, a utilização conjugada de métodos de dispersão gráfica com os agrupamentos, tem sido a alternativa mais adequada em estudos de diversidade genética.

As analises estatísticas foram realizadas por intermédio do programa computacional GENES (Cruz, 2001).

\section{RESULTADOS E DISCUSSÃO}

Baseado nos resultados das análises de variância verificou-se efeito significativo das subamostras $(\mathrm{p}<0,05)$ para todas as características avaliadas. Esses resultados indicam a existência de variabilidade genética e, consequentemente, a possibilidade de se obterem ganhos genéticos para as características em futuros trabalhos de melhoramento genético com essa espécie.

As médias dessas características foram agrupadas pelo teste de Scott-Knott a 5\% de probabilidade (Tabela 2). Destaca-se o desempenho da subamostra BGH-1739, que apresentou os maiores valores de massa fresca (MF) e comprimento do fruto (CF). Segundo Rêgo et al. (2001), essas características são importantes devido a alta correlação e o efeito direto sobre a produtividade. Já Casali et al. (1984) citam que o maior teor de matéria seca, maior comprimento do fruto, maior espessura do pericarpo e menor quantidade de sementes influenciam diretamente no aumento da firmeza do fruto. Segundo os mesmos autores, tal característica é um importante aspecto de qualidade para o consumo in natura, uma vez que frutos firmes toleram transportes a maiores distancias e podem permanecer disponíveis, possibilitando assim, os varejistas comercializarem por mais tempo.

Os altos valores de matéria fresca (MF), espessura do pericarpo (EP) e teor de sólido-solúveis (TSS); tornam a subamostra BGH-1646 interessante para a indústria alimentícia, uma vez que es- 
Tabela 1. Subamostras de Capsicum baccatum do Banco de Germoplasma de Hortaliças da UFV (BGH) estado e ano de coleta (sub-samples of Capsicum baccatum peppers from the Horticultural Germplasm Bank of from Federal University of Viçosa (BGH), State and year of collection). Viçosa, UFV, 2010.

\begin{tabular}{llllll}
\hline $\begin{array}{l}\text { Subamostra } \\
\text { do BGH }\end{array}$ & Número & $\begin{array}{c}\text { Estado e } \\
\text { ano da } \\
\text { coleta }\end{array}$ & $\begin{array}{l}\text { Subamostra } \\
\text { do BGH }\end{array}$ & Número & $\begin{array}{c}\text { Estado (país) } \\
\text { e ano da } \\
\text { coleta }\end{array}$ \\
\hline 134 & 01 & SE, 1966 & 4320 & 18 & MG, 1968 \\
181 & 02 & PE, 1966 & 4366 & 19 & MG, 1968 \\
953 & 03 & SP, 1966 & 4726 & 20 & AM, 1975 \\
1601 & 04 & RS, 1967 & 5020 & 21 & MG, 1975 \\
1607 & 05 & RS, 1967 & 5025 & 22 & ES, 1975 \\
1608 & 06 & RS, 1967 & 5102 & 23 & BA, 1975 \\
1646 & 07 & MG, 1967 & 5381 & 24 & MT, 1981 \\
1739 & 08 & SP, 1967 & 5385 & 25 & MT, 1981 \\
1761 & 09 & MG & 6026 & 26 & SP, 1985 \\
4174 & 10 & GO, 1968 & 6027 & 27 & SP, 1985 \\
4175 & 11 & MG, 1968 & 6028 & 28 & Argentina, 1985 \\
4182 & 12 & SP, 1968 & 6029 & 29 & Argentina, 1985 \\
4186 & 13 & MG, 1968 & 6207 & 30 & DF, 1986 \\
4196 & 14 & PA, 1968 & 6209 & 31 & DF, 1986 \\
4218 & 15 &,- 1968 & 6210 & 32 & DF, 1986 \\
4219 & 16 &,- 1968 & 6222 & 33 & DF, 1986 \\
4301 & 17 & GO, 1968 & 6772 & 34 & SC \\
\hline
\end{tabular}

Fonte: Texeira, 1996. sas características são determinantes no rendimento final de molhos e derivados (Casali \& Stringheta, 1984).

A análise da divergência genética por meio da distância generalizada de Mahalanobis $\left(\mathrm{D}^{2}\right)$ e o agrupamento hierárquico utilizando o método UPGMA, pode ser representado pelo dendrograma apresentado na Figura 1. No eixo X foram representadas as porcentagens das distâncias entre as subamostras e no eixo Y foram representados os 34 genótipos de Capsicum baccatum.

A interpretação do dendrograma é subjetiva e pode gerar dificuldades na tomada de decisão quanto ao número de grupos gerados, por isso é recomendado o estabelecimento de um exame visual de pontos onde ocorram altas mudanças de níveis, possibilitando a visualização dos grupos (Cruz \& Carneiro, 2006). Dessa forma, realizando o corte considerando $9 \%$ de dissimilaridade, detectou-se a formação de cinco grupos (Tabela 3). Dentre esses, destacam-se as subamostras BGH-1646, BGH-6210, BGH-1761 e BGH-1739; que possuem caracteres únicos que as separam dos

Tabela 2. Médias de matéria fresca do fruto (MF), comprimento do fruto (CF), espessura do pericarpo (EP), número de sementes por frutos (SEM) e teor de sólidos solúveis (TSS) de 34 subamostras de Capsicum baccatum, do Banco de Germoplasma de Hortaliças da Universidade Federal de Viçosa (BGH) (fruit average weight (MF), fruit length (CF), fruit diameter (EP), number of seeds per fruit (SEM) and content of soluble solids (TSS) was evaluated on 34 Capsicum baccatum subsamples from the Horticultural Germplasm Bank of from Federal University of Viçosa (BGH)). Viçosa, UFV, 2010.

\begin{tabular}{lrrrrrrrrrrr}
\hline Subamostra & \multicolumn{1}{c}{ MF } & \multicolumn{1}{c}{ CF } & EP & \multicolumn{1}{c}{ SEM } & \multicolumn{1}{c}{ TSS } & Subamostra & \multicolumn{1}{c}{ MF } & CF & EP & SEM & TSS \\
\hline $\mathbf{1 3 4}$ & $8,3 \mathrm{f}$ & $64,9 \mathrm{~d}$ & $1,3 \mathrm{e}$ & $155,2 \mathrm{a}$ & $11,9 \mathrm{a}$ & $\mathbf{4 3 2 0}$ & $7,7 \mathrm{f}$ & $30,1 \mathrm{f}$ & $3,2 \mathrm{~b}$ & $53,2 \mathrm{~d}$ & $11,8 \mathrm{a}$ \\
$\mathbf{1 8 1}$ & $7,6 \mathrm{f}$ & $73,9 \mathrm{c}$ & $1,3 \mathrm{e}$ & $102,8 \mathrm{c}$ & $10,5 \mathrm{a}$ & $\mathbf{4 3 6 6}$ & $10,0 \mathrm{f}$ & $32,8 \mathrm{f}$ & $2,3 \mathrm{c}$ & $127,0 \mathrm{~b}$ & $10,7 \mathrm{a}$ \\
$\mathbf{9 5 3}$ & $10,9 \mathrm{f}$ & $58,1 \mathrm{~d}$ & $2,2 \mathrm{c}$ & $94,6 \mathrm{c}$ & $10,8 \mathrm{a}$ & $\mathbf{4 7 2 6}$ & $8,5 \mathrm{f}$ & $44,5 \mathrm{e}$ & $2,3 \mathrm{c}$ & $73,8 \mathrm{~d}$ & $8,2 \mathrm{c}$ \\
$\mathbf{1 6 0 1}$ & $3,3 \mathrm{~h}$ & $43,2 \mathrm{e}$ & $1,4 \mathrm{e}$ & $32,0 \mathrm{e}$ & $9,3 \mathrm{~b}$ & $\mathbf{5 0 2 0}$ & $9,4 \mathrm{f}$ & $42,9 \mathrm{e}$ & $2,7 \mathrm{c}$ & $42,0 \mathrm{e}$ & $7,4 \mathrm{c}$ \\
$\mathbf{1 6 0 7}$ & $1,7 \mathrm{~h}$ & $42,2 \mathrm{e}$ & $0,8 \mathrm{f}$ & $46,6 \mathrm{~d}$ & $7,4 \mathrm{c}$ & $\mathbf{5 0 2 5}$ & $11,7 \mathrm{e}$ & $71,5 \mathrm{c}$ & $2,1 \mathrm{c}$ & $98,4 \mathrm{c}$ & $7,9 \mathrm{c}$ \\
$\mathbf{1 6 0 8}$ & $4,6 \mathrm{~g}$ & $62,3 \mathrm{~d}$ & $0,9 \mathrm{f}$ & $74,2 \mathrm{~d}$ & $7,4 \mathrm{c}$ & $\mathbf{5 1 0 2}$ & $5,1 \mathrm{~g}$ & $19,5 \mathrm{~g}$ & $2,1 \mathrm{c}$ & $73,4 \mathrm{~d}$ & $5,5 \mathrm{~d}$ \\
$\mathbf{1 6 4 6}$ & $25,7 \mathrm{~b}$ & $48,9 \mathrm{e}$ & $3,4 \mathrm{~b}$ & $148,6 \mathrm{a}$ & $8,8 \mathrm{c}$ & $\mathbf{5 3 8 1}$ & $8,7 \mathrm{f}$ & $29,8 \mathrm{f}$ & $2,6 \mathrm{c}$ & $90,0 \mathrm{c}$ & $6,5 \mathrm{~d}$ \\
$\mathbf{1 7 3 9}$ & $33,3 \mathrm{a}$ & $183,4 \mathrm{a}$ & $2,4 \mathrm{c}$ & $119,2 \mathrm{~b}$ & $8,9 \mathrm{c}$ & $\mathbf{5 3 8 5}$ & $6,5 \mathrm{~g}$ & $67,8 \mathrm{~d}$ & $1,7 \mathrm{~d}$ & $83,0 \mathrm{c}$ & $9,7 \mathrm{~b}$ \\
$\mathbf{1 7 6 1}$ & $15,7 \mathrm{~d}$ & $105,0 \mathrm{~b}$ & $2,5 \mathrm{c}$ & $55,6 \mathrm{~d}$ & $8,6 \mathrm{c}$ & $\mathbf{6 0 2 6}$ & $6,4 \mathrm{~g}$ & $71,6 \mathrm{c}$ & $1,8 \mathrm{~d}$ & $62,0 \mathrm{~d}$ & $8,0 \mathrm{c}$ \\
$\mathbf{4 1 7 4}$ & $6,0 \mathrm{~g}$ & $21,8 \mathrm{~g}$ & $2,3 \mathrm{c}$ & $53,4 \mathrm{~d}$ & $7,6 \mathrm{c}$ & $\mathbf{6 0 2 7}$ & $3,4 \mathrm{~h}$ & $46,1 \mathrm{e}$ & $1,7 \mathrm{~d}$ & $32,4 \mathrm{e}$ & $10,9 \mathrm{a}$ \\
$\mathbf{4 1 7 5}$ & $8,1 \mathrm{f}$ & $83,3 \mathrm{c}$ & $1,7 \mathrm{~d}$ & $78,0 \mathrm{~d}$ & $9,6 \mathrm{~b}$ & $\mathbf{6 0 2 8}$ & $6,1 \mathrm{~g}$ & $69,7 \mathrm{c}$ & $1,7 \mathrm{~d}$ & $81,6 \mathrm{c}$ & $11,2 \mathrm{a}$ \\
$\mathbf{4 1 8 2}$ & $7,1 \mathrm{~g}$ & $74,6 \mathrm{c}$ & $1,7 \mathrm{~d}$ & $67,8 \mathrm{~d}$ & $6,0 \mathrm{~d}$ & $\mathbf{6 0 2 9}$ & $2,6 \mathrm{~h}$ & $44,3 \mathrm{e}$ & $1,8 \mathrm{~d}$ & $15,2 \mathrm{e}$ & $11,6 \mathrm{a}$ \\
$\mathbf{4 1 8 6}$ & $4,8 \mathrm{~g}$ & $24,5 \mathrm{~g}$ & $2,8 \mathrm{c}$ & $21,0 \mathrm{e}$ & $8,4 \mathrm{c}$ & $\mathbf{6 2 0 7}$ & $10,4 \mathrm{f}$ & $75,5 \mathrm{c}$ & $1,8 \mathrm{~d}$ & $82,4 \mathrm{c}$ & $8,2 \mathrm{c}$ \\
$\mathbf{4 1 9 6}$ & $5,3 \mathrm{~g}$ & $75,4 \mathrm{c}$ & $1,5 \mathrm{~d}$ & $53,0 \mathrm{~d}$ & $9,8 \mathrm{~b}$ & $\mathbf{6 2 0 9}$ & $6,5 \mathrm{~g}$ & $58,8 \mathrm{~d}$ & $1,2 \mathrm{e}$ & $46,2 \mathrm{~d}$ & $6,5 \mathrm{~d}$ \\
$\mathbf{4 2 1 8}$ & $5,5 \mathrm{~g}$ & $80,1 \mathrm{c}$ & $1,3 \mathrm{e}$ & $68,2 \mathrm{~d}$ & $11,8 \mathrm{a}$ & $\mathbf{6 2 1 0}$ & $18,6 \mathrm{c}$ & $63,3 \mathrm{~d}$ & $2,5 \mathrm{c}$ & $108,0 \mathrm{c}$ & $7,1 \mathrm{c}$ \\
$\mathbf{4 2 1 9}$ & $3,6 \mathrm{~h}$ & $60,9 \mathrm{~d}$ & $1,1 \mathrm{e}$ & $49,8 \mathrm{~d}$ & $9,4 \mathrm{~b}$ & $\mathbf{6 2 2 2}$ & $8,2 \mathrm{f}$ & $43,9 \mathrm{e}$ & $3,1 \mathrm{~b}$ & $50,2 \mathrm{~d}$ & $7,2 \mathrm{c}$ \\
$\mathbf{4 3 0 1}$ & $12,7 \mathrm{e}$ & $39,2 \mathrm{e}$ & $4,0 \mathrm{a}$ & $86,8 \mathrm{c}$ & $7,8 \mathrm{c}$ & $\mathbf{6 7 7 2}$ & $6,9 \mathrm{~g}$ & $39,7 \mathrm{e}$ & $2,4 \mathrm{c}$ & $56,8 \mathrm{~d}$ & $6,1 \mathrm{~d}$ \\
\hline
\end{tabular}

*Grupos de médias seguidas pelas mesmas letras não diferem entre si pelo critério de Scott-Knott a 5\% de probabilidade (groups of means followed by the same letter do not differ according to the Skott Knott test $\mathrm{p}<0.05$ ). 


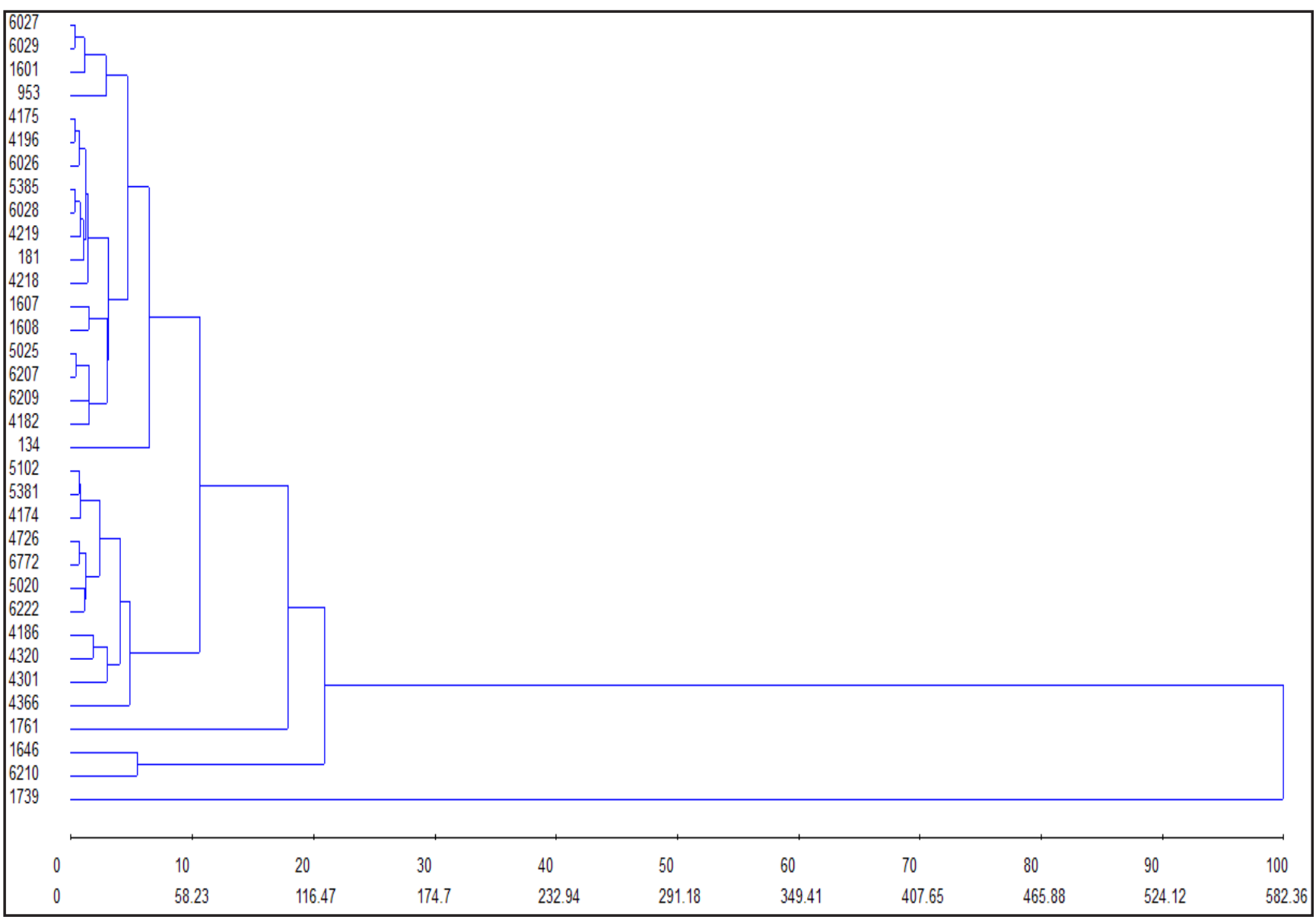

Figura 1. Dendrograma de dissimilaridade genética entre 34 subamostras de Capsicum baccatum do BGH, obtido pelo método UPGMA com base na distância generalizada de Mahalanobis $\left(\mathrm{D}^{2}\right)$ a partir de dados multicategóricos (MF, CF, EP, SEM e TSS). No eixo X encontram-se as distâncias genéticas relativas e no eixo Y encontra-se a descrição das subamostras (dendogram of genetic dissimilarity among 34 Capsicum baccatum sub-samples from BGH using de UPGMA method based on generalized distance of Mahalanobis $\left(\mathrm{D}^{2}\right)$ from multicategoric date (MF, CF, EP, SEM and TSS). On the X axis are the genetic distances and on the Y axis are the sub-samples). Viçosa, UFV, 2010.

Tabela 3. Identificação das subamostras de C. baccatum do BGH reunidos em cinco grupos pelo método de UPGMA (identification of C.baccatum sub-samples from BGH gathered into six groups by UPGMA method). Viçosa, UFV, 2010.

\begin{tabular}{ll}
\hline Grupos & \multicolumn{1}{c}{ Subamostras } \\
\hline I & $10,13,17,18,19,20,21,23,24,33,34$ \\
II & $01,02,03,04,05,06,11,12,14,15,16,22,25,26,27,28,29,30,31$ \\
III & 09 \\
IV & 07,32 \\
V & 08 \\
\hline
\end{tabular}

demais. A maior dissimilaridade foi observada entre os genótipos BGH4320 e o BGH-1739, enquanto que os mais similares foram o BGH-6029 e o BGH-6028.

Os caracteres que mais contribuíram para a formação desses grupos de divergência genética, segundo o método de Singh (1981), foram a matéria fresca $(45,6 \%)$ e o comprimento do fruto (40,9\%). Por outro lado, o número de sementes apresentou o menor valor $(0,5 \%)$, resultando em importância mínima para o agrupamento dos genótipos avaliados.

Para complementar a análise de agrupamento empregou-se a análise com base em variáveis canônicas. Nessa técnica os caracteres com maior porcentagem de variância foram empregados na dispersão dos genótipos em coordenadas cartesianas, visando sim- plificar a visualização e interpretação dos resultados (Cruz \& Carneiro, 2006). Como pode ser observado na Figura 2, o gráfico de dispersão foi coerente com o dendrograma, apresentando grande similaridade quanto à separação das subamostras em grupos.

Resultados semelhantes foram encontrados por Rêgo (2001), que analisando outros caracteres morfo-agronômicos, também observou que as subamostras BGH-1646, BGH-6210, BGH-1761, BGH-134 e BGH-1739 apresentavam características singulares, destacando-se dos demais. Dessa forma, considerando a heterose no gênero Capsicum positiva para a maioria dos caracteres estudados (Costa et al., 2002; Ikuta, 1971), a distância entre as subamostras e as altas médias de características de interesse, propõe-se a inclusão desses genótipos em programas 


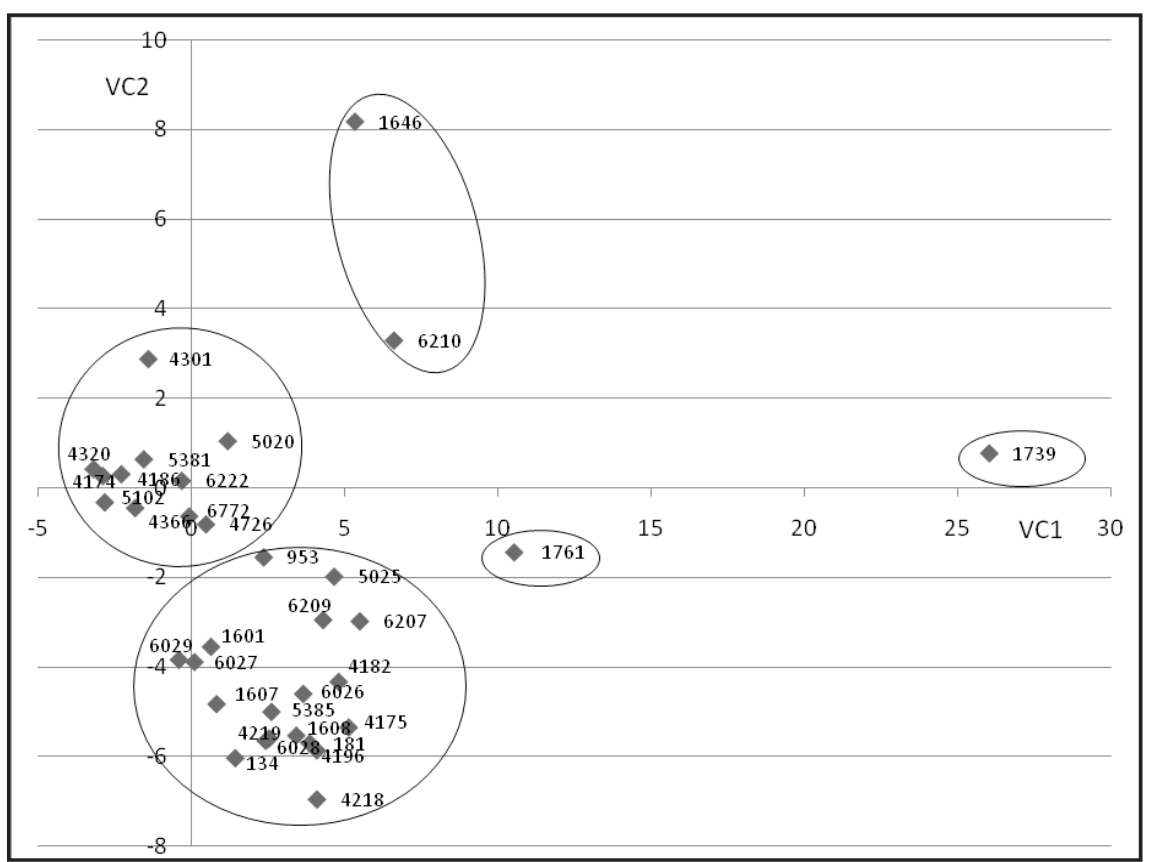

Figura 2. Dispersão gráfica de 34 subamostras de Capsicum baccatum do BGH, com base nas duas primeiras variáveis canônicas, estabelecidas pela combinação linear de cinco características agronômicas (graphic dispersion of 34 Capsicum baccatum sub-samples from $\mathrm{BGH}$, based on the first two canonical variables, established by the linear combination of five agronomic traits). Viçosa, UFV, 2010.

de melhoramento de Capsicum, visando pimenta em conserva.

Diante dos resultados observados, conclui-se que as técnicas multivariadas utilizadas foram concordantes entre si, o que permitiu a visualização da variabilidade genética entre as 34 subamostras de pimenta do BGH. Em razão disso, as subamostras aqui comentadas apresentam potencial para uso em programas de melhoramento, visando a obtenção de bons materiais para consumo in natura ou para industrialização.

\section{AGRADECIMENTOS}

À FAPEMIG pelo auxilio financeiro ao projeto.

\section{REFERÊNCIAS}

CASALI WD; PÁDUA JG; BRAZ LT. 1984. Melhoramento de pimentão e pimenta. Informe Agropecuário 10: 19-22.

CASALI WD; STRINGHETA PC. 1984. Melhoramento de pimentão e pimenta para fins industriais. Informe Agropecuário 10: 23-24.

COSTA RA; RODRIGUES R; SUDRÉ CP. 2002. Resistência à mancha bacteriana em genótipos de pimentão. Horticultura Brasileira 20: 86-89.

CRUZ CD. 2001. Programa genes (versão Windows): aplicativo computacional em genética e estatística. Viçosa-MG: UFV, 648 p.

CRUZ CD; CARNEIRO PCS. 2006. Modelos biométricos aplicados ao melhoramento genético. Viçosa-MG: UFV, v.2, 585p.

ECHER MM; FERNANDES MCA; RIBEIRO RLD; PERACCHI AL. 2002. Avaliação de genótipos de Capsicum para resistência ao ácaro branco. Horticultura. Brasileira 20: 217-221.

EMPRESA BRASILEIRA DE PESQUISA AGROPECUÁRIA. 2007. Capsicum Pimentas e Pimentões no Brasil. Brasília: Embrapa Comunicação para Transferencia de Tecnologia/ Embrapa Hortaliças. Disponível em $:<$ http://www.cnph.embrapa.br $>$ Acessado em 28 de fevereiro de 2010.

GEPTS P. 2006. Plant genetic resources conservation and utilization: the accomplishments and future of a societal insurance policy. Crop Science 46: 2278-2292.

GONÇALVES LSA; RODRIGUES R; SUDRÉ CP; BENTO CS; MOULIN MM; ARAÚJO ML; DAHER RF; PEREIRA TNS; PEREIRA MG. 2008. Divergência genética em tomate estimada por marcadores RAPD em comparação com descritores multicategóricos. Horticultura Brasileira 26: 364-370.

IKUTA H. 1971. Ensaio de híbridos F1, F2 e variedades resistentes a vírus de pimentão (Capsicum annum L.). Revista de Olericultura 11: 64 .

IPGRI. 1995. Descriptores para Capsicum (Capsicum spp). Roma: IPGRI, 51.

LANNES SD; FINGER FL; SCHUELTER DR; CASALI VWD. 2007. Growth and quality of Brazilian accessions of Capsicum chinense fruits. Scientia Horticulturae 112: 266-270.

PICKERSGILL B. 1997. Genetic resources and breeding of Capsicum spp. Euphytica 96: 129-133.

RAO CR. 1952. An advanced statistical method in biometric research. New York: John Wiley \& Sons. 390p.

RÊGO ER. 2001. Diversidade, herança e capacidade combinatória em pimenta (Capsicum baccatum). Viçosa: UFV, (Tese doutorado).

SINGH D. 1981. The relative importance of characters affecting genetic divergence. The Indian J. of Genet. and Plant Breed 41: 237-245.

WAGNER CM. 2003. Variabilidade e base genética da pungência e de caracteres do fruto: Implicações no melhoramento de uma população de Capsicum annum L. Piracicaba: ESALQ. 104p (Tese doutorado). 